\title{
On the solution of linear and nonlinear partial differential equations: applications of local fractional Sumudu variational method
}

\author{
Badr S Alkahtania, Obaid J. Algahtania , Pranay Goswami ${ }^{b, *}$ \\ ${ }^{a}$ Mathematics Department, College of Science, King Saud University, Riyadh 11989, Saudi Arabia. \\ ${ }^{b}$ School of Liberal Studies, Ambedkar University Delhi, Delhi-11006, India.
}

\begin{abstract}
In this paper, the local fractional Sumudu variational iteration method is being used to investigate the solutions of partial differential equations containing the local fractional derivatives. The present technique is the combination of the local fractional Sumudu transform and fractional variational iteration method. Three illustrative examples are given to demonstrate the efficiency of the method. (C)2016 all rights reserved.

Keywords: Modified Riemann-Liouville derivative, local fractional derivative, fractional variational iteration method, fractional differential equations, local fractional Sumudu transform.

2010 MSC: 26A33, 35A22, 33E12, 35R11, 65L10.
\end{abstract}

\section{Introduction}

Fractional calculus and local fractional calculus are very popular in the fields of physics and engineering $([2,11,13,17,23])$. Most of the phenomena are represented in terms of partial fractional differential equations. The solutions of these equations are very tempting due to process of finding and level of reliability they holds. Another attracting thing is that the number of techniques is presented in literature for solving such equations ([1, 3-5, 7, 10, 12, 14, 15]). The variational iteration method $([8,9])$ is the most popular method that enriched the solutions of partial differential equations due

\footnotetext{
*Corresponding author

Email addresses: alhaghog@gmail.com (Badr S Alkahtani), obalgahtani@ksu.edu.sa (Obaid J. Algahtani), pranaygoswami83@gmail.com (Pranay Goswami)
} 
to its easy handling and flexibility $([16,19,20,25,26])$. Nowadays the combination of variational iteration method and other analytical methods is attracting the researchers and earthen out more and more interesting ways to solve these types of equations $([\underline{6}, 24])$. The local fractional Sumudu variational iteration method is very new and fresh technique for these equations. The local fractional variational iteration method is derived by the combination of the local fractional Sumudu transform [18] and the fractional variational iteration method. This technique is being popular day by day due to its freshness and easy implementation with high level of efficiency.

In this article, our aim is to use the fractional variational iteration method and the local fractional Sumudu transform to develop an iteration formula to solve the linear and nonlinear local fractional partial differential equations with the local fractional derivatives.

\section{Definitions and mathematical preliminaries}

Definition 2.1 ([21, 22]). The partition of interval $[a, b]$ is denoted as $\left(t_{j}, t_{j+1}\right), j=0, \ldots, N-1, t_{0}=$ $a$ and $t_{N}=b$ with $\Delta t_{j}=t_{j+1}-t_{j}$ and $\Delta t=\max \left\{\Delta t_{0}, \Delta t_{1} \ldots\right\}$. Local fractional integral of $f(x)$ in the interval $[a, b]$ is given by

$$
{ }_{a} I_{b}^{(\alpha)} f(x)=\frac{1}{\Gamma(1+\alpha)} \int_{a}^{b} f(t)(d t)^{\alpha}=\frac{1}{\Gamma(1+\alpha)} \lim _{\Delta t \rightarrow 0} \sum_{j=0}^{N-1} f\left(t_{j}\right)\left(\Delta t_{j}\right)_{\alpha} .
$$

Definition $2.2([21,22])$. The local fractional derivative of $f(x) \in C_{\alpha}(a, b)$ of order $\alpha$ at $x=x_{0}$ is defined as

$$
f^{(\alpha)}\left(x_{0}\right)=\left.\frac{d^{\alpha}}{d x^{\alpha}} f(x)\right|_{x=x_{0}}=\lim _{x \rightarrow x_{0}} \frac{\Delta^{\alpha}\left(f(x)-f\left(x_{0}\right)\right)}{\left(x-x_{0}\right)^{\alpha}},
$$

where $\Delta^{\alpha}\left(f(x)-f\left(x_{0}\right)\right) \cong \Gamma(\alpha+1) f(x)-f\left(x_{0}\right)$.

Definition 2.3. The local fractional Sumudu transform of $f(t)$ of order $\alpha$ is defined as [18]

$$
S_{\alpha}[f(t)]=G_{\alpha}(u)=\frac{1}{\Gamma(1+\alpha)} \int_{0}^{\infty} E_{\alpha}\left(-u^{\alpha} t^{\alpha}\right) \frac{f(t)}{z^{\alpha}}(d t)^{\alpha}, \quad 0<\alpha \leq 1,
$$

where $x \in C$ and $E_{\alpha}(x)$ is Mittage Leffler function $\sum_{k=0}^{\infty}\left(\frac{x^{k}}{a k !}\right)$. And the inverse Sumudu transform is defined as

$$
S_{\alpha}^{-1}\{f(u)\}=f(t), \quad 0<\alpha \leq 1 .
$$

Using the above definition of the local fractional Sumudu transform the following result can easily be obtained [18]

$$
\begin{aligned}
S_{\alpha}\left\{f^{\alpha}(t)\right\} & =\frac{G_{\alpha}(u)-f(0)}{u^{\alpha}} \\
S_{\alpha}\left\{f^{n \alpha}(t)\right\} & =\frac{1}{u^{n \alpha}}\left[G_{\alpha}(u)-\sum_{k=0}^{n-1} u^{k \alpha} f^{(k \alpha)}(0)\right] .
\end{aligned}
$$

\section{The local fractional Sumudu variational iteration method}

This section introduces the idea of fractional Sumudu variational method for the following fractional partial differential equation

$$
D_{0+}^{m \alpha} v(x, t)=L_{\alpha}(v(x, t))+N_{\alpha}(v(x, t))+f(x, t),
$$

where $0<\alpha \leq 1, x, t \geq 0, L_{\alpha}$ is a linear operator, $N_{\alpha}$ is nonlinear operator, and $f$ is a source term. 
Taking local fractional Sumudu transform of (3.1) both the sides w.r.t. ' $t$ ',

$$
\frac{1}{u^{m \alpha}}\left[G_{\alpha}(u)-\sum_{k=0}^{m-1} u^{k \alpha} v^{(k \alpha)}(x, 0)\right]=S_{\alpha}\left[L_{\alpha}(v(x, t))+N_{\alpha}(v(x, t))+f(x, t)\right] .
$$

For an algebraic equation the iterative formula can be constructed as

$$
x_{n+1}=x_{n}+\lambda^{\alpha} f\left(x_{n}\right) .
$$

The optimality condition for the extreme $\frac{\delta x_{n+1}}{\delta x_{n}}=0$, leads to

$$
\lambda^{\alpha}=-\frac{1}{f^{\prime}\left(x_{n}\right)},
$$

where $\delta$ is the classical variational operator.

By the formula (3.2), we get the iterative formula for equation (3.1) as follows,

$$
\begin{aligned}
G_{\alpha} v_{n+1}(x, u)=G_{\alpha} v_{n}(x, u) & +\lambda^{\alpha}\left\{\frac{1}{u^{m \alpha}}\left[G_{\alpha} v_{n}(x, u)-\sum_{k=0}^{m-1} u^{k \alpha} v^{(k \alpha)}(x, 0)\right]\right. \\
& \left.-S_{\alpha}\left[L_{\alpha}\left(v_{n}(x, t)\right)+N_{\alpha}\left(v_{n}(x, t)\right)+f(x, t)\right]\right\} .
\end{aligned}
$$

Put $\lambda^{\alpha}=-u^{m \alpha}$, the Lagrange multiplier in (3.3)

$$
G_{\alpha} v_{n+1}(x, u)=+\left\{\left[\sum_{k=0}^{m-1} u^{k \alpha} v^{(k \alpha)}(x, 0)\right]+u^{m \alpha} S_{\alpha}\left[L_{\alpha}\left(v_{n}(x, t)\right)+N_{\alpha}\left(v_{n}(x, t)\right)+f(x, t)\right]\right\} .
$$

Taking the inverse fractional Sumudu transform, we arrived at

$$
v_{n+1}(x, t)=S_{\alpha}^{-1}\left\{\left[\sum_{k=0}^{m-1} u^{k \alpha} v^{(k \alpha)}(x, 0)\right]+u^{m \alpha} S_{\alpha}\left[L_{\alpha}\left(v_{n}(x, t)\right)+N_{\alpha}\left(v_{n}(x, t)\right)+f(x, t)\right]\right\} .
$$

This is the iterative formula for equation (3.1).

Example 3.1. The generalized linear fractional partial differential equation for $0 \leq x \leq 1,0 \leq t \leq$ $1,0<\alpha \leq 1,0<\beta \leq 1$ is as follows

$$
\frac{\partial^{\alpha}}{\partial x^{\alpha}} v(x, t)+x^{\beta} \frac{\partial^{\beta} v(x, t)}{\partial x^{\beta}}+\frac{\partial^{2 \beta} v(x, t)}{\partial x^{2 \beta}}=2\left(t^{\alpha}+x^{2 \beta}+1\right),
$$

with initial condition

$$
v(x, 0)=x^{2 \beta} .
$$

Proof. By using the iterative formula (3.4) derived above, we get the following iterative formula for equation 3.5 as

$$
v_{n+1}(x, t)=S_{\alpha}^{-1}\left[\sum_{k=0}^{n-1}\left[u^{k \alpha} v^{k \alpha}(x, 0)\right]+u^{\alpha} S_{\alpha}\left(2\left(t^{\alpha}+x^{2 \beta}+1\right)-x^{\beta} \frac{\partial^{\beta} v_{n}}{\partial x^{\beta}}-\frac{\partial^{2 \beta} v_{n}}{\partial x^{2 \beta}}\right)\right] .
$$


Let us start from the initial iteration, which is given as

$$
v_{0}(x, t)=S_{\alpha}^{-1}\left[\sum_{k=0}^{n-1}\left[u^{k \alpha} v^{k \alpha}(x, 0)\right]+u^{\alpha} S_{\alpha}\left(2\left(t^{\alpha}+x^{2 \beta}+1\right)\right)\right] .
$$

Solving the second term of above equation by taking the local fractional Sumudu transform and using the initial condition,

$$
v_{0}(x, t)=x^{2 \beta}+S_{\alpha}^{-1}\left[u^{\alpha}\left(2 u^{\alpha} \Gamma(1+\alpha)+x^{2 \beta}+1\right)\right] .
$$

After taking the local fractional Sumudu transform in (3.7), we get the initial iteration $v_{0}(x, t)$ as

$$
v_{0}(x, t)=x^{2 \beta}+\frac{2 t^{2 \alpha}}{\Gamma(1+2 \alpha)} \Gamma(1+\alpha)+x^{2 \beta} \frac{2 t^{\alpha}}{\Gamma(1+\alpha)}+\frac{t^{\alpha}}{\Gamma(1+\alpha)} .
$$

Now for $v_{1}(x, t)$, we have the iterative formula by using (3.6) with $n=0$ as follows

$$
v_{1}(x, t)=S_{\alpha}^{-1}\left[\sum_{k=0}^{m-1}\left[u^{k \alpha} v^{k \alpha}(x, 0)\right]+u^{\alpha} S_{\alpha}\left(2\left(t^{\alpha}+x^{2 \beta}+1\right)-x^{\beta} \frac{\partial^{\beta} v_{0}}{\partial x^{\beta}}-\frac{\partial^{2 \beta} v_{0}}{\partial x^{2 \beta}}\right)\right] .
$$

Or we can write equation $(3.8)$ as

$$
v_{1}(x, t)=v_{0}(x, t)+S_{\alpha}^{-1}\left[u^{\alpha} S_{\alpha}\left(-x^{\beta} \frac{\partial^{\beta} v_{0}}{\partial x^{\beta}}-\frac{\partial^{2 \beta} v_{0}}{\partial x^{2 \beta}}\right)\right] .
$$

Putting the value of $v_{0}(x, t)$ in 3.9$)$ and solving the derivative terms we get

$$
\begin{aligned}
v_{1}(x, t)= & x^{2 \beta}+\frac{2 t^{2 \alpha}}{\Gamma(1+2 \alpha)} \Gamma(1+\alpha)+x^{2 \beta} \frac{2 t^{\alpha}}{\Gamma(1+\alpha)}+\frac{2 t^{\alpha}}{\Gamma(1+\alpha)} \\
& +S_{\alpha}^{-1}\left[u^{\alpha} S_{\alpha}\left(-x^{\beta}\left(2 \beta x^{\beta}+4 \beta x^{\beta} \frac{t^{\alpha}}{\Gamma(1+\alpha)}\right)-\left(2 \beta^{2}+4 \beta^{2} \frac{t^{\alpha}}{\Gamma(1+\alpha)}\right)\right)\right] .
\end{aligned}
$$

Applying the local fractional Sumudu transform, we have

$$
\begin{aligned}
v_{1}(x, t)= & x^{2 \beta}+\frac{2 t^{2 \alpha}}{\Gamma(1+2 \alpha)} \Gamma(1+\alpha)+x^{2 \beta} \frac{2 t^{\alpha}}{\Gamma(1+\alpha)}+\frac{2 t^{\alpha}}{\Gamma(1+\alpha)} \\
& +S_{\alpha}^{-1}\left[u^{\alpha}\left(-x^{\beta}\left(2 \beta x^{\beta}+4 \beta x^{\beta} u^{\alpha}\right)-\left(2 \beta^{2}+4 \beta^{2} u^{\alpha}\right)\right)\right] .
\end{aligned}
$$

Now taking the inverse local fractional Sumudu transform we get $v_{1}(x, t)$ as follows:

$$
\begin{aligned}
v_{1}(x, t)= & x^{2 \beta}+\frac{2 t^{2 \alpha}}{\Gamma(1+2 \alpha)} \Gamma(1+\alpha)+x^{2 \beta} \frac{2 t^{\alpha}}{\Gamma(1+\alpha)}+\frac{2 t^{\alpha}}{\Gamma(1+\alpha)} \\
& +\left[\left(-x^{\beta}\left(2 \beta x^{\beta} \frac{t^{\alpha}}{\Gamma(1+\alpha)}+4 \beta x^{\beta} \frac{t^{2 \alpha}}{\Gamma(1+2 \alpha)}\right)-\left(2 \beta^{2} \frac{t^{\alpha}}{\Gamma(1+\alpha)}+4 \beta^{2} \frac{t^{2 \alpha}}{\Gamma(1+2 \alpha)}\right)\right)\right] .
\end{aligned}
$$

Similarly we can find

$$
\begin{aligned}
v_{2}(x, t)= & x^{2 \beta}+\frac{2 t^{2 \alpha}}{\Gamma(1+2 \alpha)} \Gamma(1+\alpha)+x^{2 \beta} \frac{2 t^{\alpha}}{\Gamma(1+\alpha)}+\frac{2 t^{\alpha}}{\Gamma(1+\alpha)} \\
& +\left[\left(-x^{\beta}\left(2 \beta x^{\beta} \frac{t^{\alpha}}{\Gamma(1+\alpha)}+4 \beta x^{\beta} \frac{t^{2 \alpha}}{\Gamma(1+2 \alpha)}\right)-\left(2 \beta^{2} \frac{t^{\alpha}}{\Gamma(1+\alpha)}+4 \beta^{2} \frac{t^{2 \alpha}}{\Gamma(1+2 \alpha)}\right)\right)\right] \\
& +x^{2 \beta} \frac{4 \beta^{2} t^{2 \alpha}}{\Gamma(1+2 \alpha)}+8 \beta^{2} x^{2 \beta} \frac{t^{3 \alpha}}{\Gamma(1+3 \alpha)}+4 \beta^{3} \frac{t^{2 \alpha}}{\Gamma(1+2 \alpha)}+8 \beta^{3} \frac{t^{3 \alpha}}{\Gamma(1+3 \alpha)} .
\end{aligned}
$$


Then general term of successive approximation is given by

$$
\begin{aligned}
v_{n}(x, t)= & x^{2 \beta}+\frac{2 t^{2 \alpha}}{\Gamma(1+2 \alpha)} \Gamma(1+\alpha)+\sum_{k=1}^{n}(-1)^{k}(2 \beta)^{k} \frac{t^{k \alpha}}{\Gamma(1+k \alpha)}\left(x^{2 \beta}+\beta\right) \\
& +\sum_{k=0}^{n}(-1)^{k} 2^{k+1} \beta^{k} \frac{t^{(k+1) \alpha}}{\Gamma(1+(k+1) \alpha)}\left(x^{2 \beta}+\beta\right)
\end{aligned}
$$

Example 3.2. Consider the following local fractional nonlinear transport equation

$$
\frac{\partial^{\alpha}}{\partial t^{\alpha}} v(x, t)-a(x, t) \frac{\partial^{2 \alpha}}{\partial x^{2 \alpha}} v(x, t)-\frac{b(x, t) v(x, t)}{2} \frac{\partial^{\alpha}}{\partial x^{\alpha}} v(x, t)=0, t>0,1<\alpha \leq 2, x \in \Re,
$$

with initial condition

$$
v(x, 0)=\frac{x^{\alpha}}{\Gamma(1+\alpha)}, \text { where } a(x, t)=1 \text { and } b(x, t)=2 .
$$

Proof. By using the iterative formula derived from (3.4) above, we get the following iterative formula for equation 3.10 .

$$
v_{n+1}(x, t)=S_{\alpha}^{-1}\left[\sum_{k=0}^{n-1}\left[u^{k \alpha} v^{k \alpha}(x, 0)\right]+u^{\alpha} S_{\alpha}\left(\frac{\partial^{2 \alpha} v_{n}(x, t)}{\partial x^{2 \alpha}}+v_{n}(x, t) \frac{\partial^{\alpha} v_{n}(x, t)}{\partial x^{\alpha}}\right)\right] .
$$

The initial iteration $v_{0}(x, t)$ is given as follows

$$
v_{0}(x, t)=S_{\alpha}^{-1}\left[\sum_{k=0}^{n-1}\left[u^{k \alpha} v^{k \alpha}(x, 0)\right]\right] .
$$

Using initial values we get

$$
v_{0}(x)=S_{\alpha}^{-1}\left[\frac{x^{\alpha}}{\Gamma(1+\alpha)}\right] .
$$

Taking the inverse local fractional Sumudu transform in 3.12 , we get

$$
v_{0}(x, t)=\frac{x^{\alpha}}{\Gamma(1+\alpha)} .
$$

Now, for $v_{1}(x, t)$ we can find the iterative formula as follows by putting $n=0$ in (3.11)

$$
\begin{aligned}
& v_{1}(x, t)=\frac{x^{\alpha}}{\Gamma(1+\alpha)}+S_{\alpha}^{-1}\left[u^{\alpha} S_{\alpha}\left(\frac{\partial^{2 \alpha} v_{0}(x, t)}{\partial x^{2 \alpha}}+v_{0}(x, t) \frac{\partial^{\alpha} v_{n}(x, t)}{\partial x^{\alpha}}\right)\right], \\
& v_{1}(x, t)=\frac{x^{\alpha}}{\Gamma(1+\alpha)}+S_{\alpha}^{-1}\left[u^{\alpha} S_{\alpha}\left(\frac{x^{\alpha}}{\Gamma(1+\alpha)} \cdot \frac{1}{\Gamma(1+\alpha)}\right)\right], \\
& v_{1}(x, t)=\frac{x^{\alpha}}{\Gamma(1+\alpha)}+S_{\alpha}^{-1}\left[u^{\alpha}\left(\frac{x^{\alpha}}{\Gamma(1+\alpha)} \cdot \frac{1}{\Gamma(1+\alpha)}\right)\right], \\
& v_{1}(x, t)=\frac{x^{\alpha}}{\Gamma(1+\alpha)}+\frac{t^{\alpha}}{\Gamma(1+\alpha)} \frac{x^{\alpha}}{\Gamma(1+\alpha)},
\end{aligned}
$$




$$
v_{1}(x, t)=\frac{x^{\alpha}}{\Gamma(1+\alpha)}\left(1+\frac{t^{\alpha}}{\Gamma(1+\alpha)}\right) .
$$

Similiarly, we can find other iterations $v_{2}(x, t)$ as

$$
\begin{aligned}
v_{2}(x, t)= & \frac{x^{\alpha}}{\Gamma(1+\alpha)}+S_{\alpha}^{-1}\left[u^{\alpha} S_{\alpha}\left(\frac{\partial^{2 \alpha} v_{1}(x, t)}{\partial x^{2 \alpha}}+v_{1}(x, t) \frac{\partial^{\alpha} v_{1}(x, t)}{\partial x^{\alpha}}\right)\right], \\
v_{2}(x, t)= & \frac{x^{\alpha}}{\Gamma(1+\alpha)}+S_{\alpha}^{-1}\left[u^{\alpha} S_{\alpha}\left(\frac{x^{\alpha}}{\Gamma(1+\alpha)}\left(1+\frac{t^{\alpha}}{\Gamma(1+\alpha)}\right)\left(1+\frac{t^{\alpha}}{\Gamma(1+\alpha)}\right)\right)\right], \\
v_{2}(x, t)= & \frac{x^{\alpha}}{\Gamma(1+\alpha)}+S_{\alpha}^{-1}\left[u^{\alpha}\left(\frac{x^{\alpha}}{\Gamma(1+\alpha)}+2 u^{\alpha} \frac{x^{\alpha}}{\Gamma(1+\alpha)}+\frac{u^{2 \alpha} \Gamma(1+2 \alpha)}{\Gamma(1+\alpha) \Gamma(1+\alpha)} \frac{x^{\alpha}}{\Gamma(1+\alpha)}\right)\right], \\
v_{2}(x, t)= & \frac{x^{\alpha}}{\Gamma(1+\alpha)}+\frac{t^{\alpha}}{\Gamma(1+\alpha)} \frac{x^{\alpha}}{\Gamma(1+\alpha)}+2 \frac{t^{2 \alpha}}{\Gamma(1+2 \alpha)} \frac{x^{\alpha}}{\Gamma(1+\alpha)} \\
& +\frac{t^{3 \alpha}}{\Gamma(1+3 \alpha)} \frac{\Gamma(1+2 \alpha)}{\Gamma(1+\alpha) \Gamma(1+\alpha)} \frac{x^{\alpha}}{\Gamma(1+\alpha)} .
\end{aligned}
$$

Example 3.3. Consider the following local fractional nonlinear Fokker- Plank equation

$$
\partial^{\alpha} \partial t^{\alpha} v(x, t)=a(x, v) \frac{\partial^{2 \alpha}}{\partial x^{2 \alpha}} v(x, t)-b(x, v) \frac{\partial^{\alpha}}{\partial x^{\alpha}} v(x, t), \quad t>0,1<\alpha \leq 2, x \in \Re
$$

with initial condition

$$
v(x, 0)=E_{\alpha}\left(x^{\alpha}\right), \text { where } a(x, v)=v(x, t) \text { and } b(x, v)=v(x, t) .
$$

Proof. By using the iterative formula derived from (3.4) above, we get the following iterative formula for $(3.13)$ as

$$
v_{n+1}(x, t)=S_{\alpha}^{-1}\left[\sum_{k=0}^{n-1}\left[u^{k \alpha} v^{k \alpha}(x, 0)\right]+u^{\alpha} S_{\alpha}\left(v_{n}(x, t) \frac{\partial^{2 \alpha} v_{n}(x, t)}{\partial x^{2 \alpha}}-v_{n}(x, t) \frac{\partial^{\alpha} v_{n}(x, t)}{\partial x^{\alpha}}\right)\right] .
$$

The initial iteration is given as

$$
\begin{aligned}
& v_{0}(x, t)=S_{\alpha}^{-1}\left[\sum_{k=0}^{n-1}\left[u^{k \alpha} v^{k \alpha}(x, 0)\right]\right], \\
& v_{0}(x, t)=S_{\alpha}^{-1}\left[E_{\alpha}\left(x^{\alpha}\right)\right]=E_{\alpha}\left(x^{\alpha}\right) .
\end{aligned}
$$

By applying same calculation process used in previous examples we get the following iteration $v_{1}(x, t)$ :

$$
\begin{aligned}
& v_{1}(x, t)=E_{\alpha}\left(x^{\alpha}\right)+S_{\alpha}^{-1}\left[u^{\alpha} S_{\alpha}\left(v_{0}(x, t) \frac{\partial^{2 \alpha} v_{0}(x, t)}{\partial x^{2 \alpha}}-v_{0}(x, t) \frac{\partial^{\alpha} v_{0}(x, t)}{\partial x^{\alpha}}\right)\right], \\
& v_{1}(x, t)=E_{\alpha}\left(x^{\alpha}\right) .
\end{aligned}
$$

In the same manner as above, the rest of the approximative solutions are presented as follows

$$
v_{2}(x, t)=v_{3}(x, t)=\cdots=v_{n}(x, t)=E_{\alpha}\left(x^{\alpha}\right) .
$$

The closed form solution can be written as follows

$$
v(x, t)=\lim _{n \rightarrow \infty} v_{n}(x, t)=E_{\alpha}\left(x^{\alpha}\right) .
$$




\section{References}

[1] K. Al-Khaled, S. Momani, An approximate solution for a fractional diffusion-wave equation using the decomposition method, Appl. Math. Comput., 165 (2005), 473-483. 1

[2] D. Baleanu, K. Diethelm, E. Scalas, J. J. Trujillo, Fractional calculus, Models and numerical methods, Series on Complexity, Nonlinearity and Chaos, World Scientific Publishing Co. Pte. Ltd., Hackensack, NJ, (2012). 1

[3] J. Chen, F. Liu, V. Anh, Analytical solution for the time-fractional telegraph equation by the method of separating variables, J. Math. Anal. Appl., 338 (2008), 1364-1377. 1

[4] L. Debnath, D. D. Bhatta, Solutions to few linear fractional inhomogeneous partial differential equations in fluid mechanics, Fract. Calc. Appl. Anal., 7 (2004), 21-36.

[5] M. Dehghan, F. Shakeri, Approximate solution of a differential equation arising in astrophysics using the variational iteration method, New Astron., 13 (2008), 53-59. 1

[6] D. D. Ganji, A. Sadighi, Application of homotopy-perturbation and variational iteration methods to nonlinear heat transfer and porous media equations, J. Comput. Appl. Math., 207 (2007), 24-34. 1 .

[7] A. Hanyga, Multidimensional solutions of time-fractional diffusion-wave equations, R. Soc. Lond. Proc. Ser. A Math. Phys. Eng. Sci., 458 (2002), 933-957. 1

[8] J. H. He, Variational iteration method - a kind of non-linear analytical technique: some examples, Int. J. Nonlinear Mech., 34 (1999), 699-708 . 1

[9] J. H. He, Variational iteration method-some recent results and new interpretations, J. Comput. Appl. Math., 207 (2007), 3-17. 1

[10] H. Jafari, H. K. Jassim, Numerical solutions of telegraph and Laplace equations on Cantor sets using local fractional Laplace decomposition method, Int. J. Adv. Appl. Math. Mech., 2 (2015), 144-151. 1

[11] A. A. Kilbas, H. M. Srivastava, J. J. Trujillo, Theory and applications of fractional differential equations, North-Holland Mathematics Studies, Elsevier Science B.V., Amsterdam, (2006). 1

[12] F. Mainardi, Y. Luchko, G. Pagnini, The fundamental solution of the space-time fractional diffusion equation, Fract. Calc. Appl. Anal., 4 (2001), 153-192. 1

[13] K. S. Miller, B. Ross, An introduction to the fractional calculus and fractional differential equations, A Wiley-Interscience Publication, John Wiley \& Sons, Inc., New York, (1993). 1

[14] S. Momani, An explicit and numerical solutions of the fractional KdV equation, Math. Comput. Simulation, 70 (2005), 110-118. 1

[15] Z. Odibat, S. Momani, Approximate solutions for boundary value problems of time-fractional wave equation, Appl. Math. Comput., 181 (2006), 767-774. 1

[16] Z. Odibat, S. Momani, The variational iteration method: an efficient scheme for handling fractional partial differential equations in fluid mechanics, Comput. Math. Appl., 58 (2009), 2199-2208. 1

[17] K. B. Oldham, J. Spanier, The fractional calculus, Theory and applications of differentiation and integration to arbitrary order, With an annotated chronological bibliography by Bertram Ross, Mathematics in Science and Engineering, Academic Press, New York-London, (1974). 1

[18] H. M. Srivastava, A. K. Golmankhaneh, D. Baleanu, X. J. Yang, Local fractional Sumudu transform with application to IVPs on Cantor sets, Abstr. Appl. Anal., 2014 (2014), 7 pages. 1, 2.3, 2

[19] N. H. Sweilam, M. M. Khader, Variational iteration method for one dimensional nonlinear thermoelasticity, Chaos Solitons Fractals, 32 (2007), 145-149. 1

[20] V. Turut, N. Güzel, On solving partial differential equations of fractional order by using the variational iteration method and multivariate Padé approximations, Eur. J. Pure Appl. Math., 6 (2013), 147-171. 1

[21] X. J. Yang, Local fractional functional analysis and its applications, Asian Academic publisher Limited, Hong Kong, (2011). 2.1.2.2

[22] X. J. Yang, Local fractional integral transforms, Prog. Nonlinear Sci., 4 (2011), 12-25. 2.1. 2.2

[23] X. J. Yang, Advanced local fractional calculus and its applications, World Sci., New York, (2012). 1

[24] A. M. Yang, J. Li, H. M. Srivastava, G. N. Xie, X. J. Yang, Local fractional Laplace variational iteration method for solving linear partial differential equations with local fractional derivative, Discrete Dyn. Nat. Soc., 2015 (2015), 9 pages. 1

[25] A. M. Wazwaz, The variational iteration method: a powerful scheme for handling linear and nonlinear diffusion equations, Comput. Math. Appl., 54 (2007), 933-939. 1

[26] A. M. Wazwaz, The variational iteration method for rational solutions for $K d V, K(2,2)$, Burgers, and cubic Boussinesq equations, J. Comput. Appl. Math., 207 (2007), 18-23 1 